To be published in Optics Letters:

Title: $\quad$ Compact linear polarization spectrometer based on radiation mode shaped in-fiber diffract Authors: Huabao Qin, Qinyun He,Yarien Moreno,Zhikun Xing,Xi Guo,Zhijun Yan,Qizhen Sun,Kaimi Accepted: $\quad 30$ September 19

Posted $\quad 30$ September 19

Doc. ID: 376282 


\title{
Compact linear polarization spectrometer based on radiation mode shaped in-fiber diffraction grating
}

\author{
Huabao QIn, ${ }^{1}$ QInyun He, ${ }^{1}$ Yarien Moreno, ${ }^{1,4}$ ZhIKUn XING, ${ }^{1}$ XI GuO, ${ }^{1}$ \\ ZHIJUN YAN, ${ }^{1,2,3, *}$ QIZHEN SUN, ${ }^{1,2}$ KaIMING ZHOU, ${ }^{3}$ DEMING LIU ${ }^{1,2}$ AND LIN \\ ZHANG $^{3}$ \\ ${ }^{1}$ The School of Optical and Electronic Information, National Engineering Laboratory for Next Generation Internet Access System, Huazhong \\ University of Science and Technology, Wuhan 430074, Hubei, P. R. China \\ ${ }^{2}$ Wuhan National Laboratory for Optoelectronics (WNLO), Huazhong University of Science and Technology (HUST), 430074 Wuhan, China \\ ${ }^{3}$ Aston Institute of Photonic Technologies, Aston University, Birmingham, UK, B4 TET \\ ${ }^{4}$ Faculty of Engineering, University of Panama, Panama City, Panama \\ *Corresponding author: yanzhijun@gmail.com
}

Received XX Month XXXX; revised XX Month, XXXX; accepted XX Month XXXX; posted XX Month XXXX (Doc. ID XXXXX); published XX Month XXXX

We proposed a compact linear polarization spectrometer based on the in-fiber polarization dependent diffraction grating. The beam profile of radiated light of the grating has been shaped to be a Gaussian profile to improve the performance of the spectrometer, where the size of the focused light spot has been reduced from 44um to $33 \mathrm{um}$ with the shaped radiation mode of the grating. Based on the experimental results, the proposed spectrometer could achieve $0.05 \mathrm{~nm}$ resolution and $115 \mathrm{~nm}$ wavelength responding range from $1495 \mathrm{~nm}$ to $1610 \mathrm{~nm}$. To verify the performance of the proposed fiber spectrometer, we have measured the transmission spectra of an excessively tilted fiber grating (Ex-TFG), which has a pair of orthogonal polarization transmission spectra. Compared with the traditional measuring method, the proposed fiber spectrometer has integrated the polarizing and spectral analyzing functions in the measuring system and achieved the polarization sensitive spectral analysis, which showed good wavelength consistency and perfect polarization characteristics. (C) 2018 Optical Society of America

http://dx.doi.org/10.1364/OL.99.099999

Polarization spectral analysis (PSA) technique is a novel and important method for noninvasive in-vivo imaging [1-6], which has been widely applied in polarization sensitive optical coherence tomography (PS-OCT) and bioimaging areas because that the birefringence of the biological tissues makes the imaging results show a strong polarization dependency. Compared to the traditional OCT technique, the introduction of the polarization could provide additional and detailed information of the structures of biological tissues and further improve the quality of the images.
Many valuable works of PSA have been presented in the recent years, which have verified the significance of the application of polarization in the optical systems. In 2010, a single-camera PSOCT has been proposed by T. Schmoll et al. for high-quality and high-speed in-vivo nail fold imaging based on the differentiation between two orthogonal polarization channels through spatial modulation [2]. And in 2011, K. Kim et al. have proposed a PS optical frequency domain imaging system and imaged the normal and cancer tissues by illuminating the tissues with the light composed of two orthogonal polarization states, where the difference between these two tissues became more distinguishable with the introduction of the polarization information [3]. M. Zhao et al. have proposed a PS-OCT system based on the single camera and phase unwrapping method for retinal imaging and E. Gotzinger et al. have imaged the human retina with two polarization channels, where two spectrometers have been applied in the system for each channel [4-5]. H. Wang et al. developed a PS-OCT based on the polarization maintaining fiber (PMF) to investigate the structure the brain, where the results showed that PS-OCT had a stronger ability to identify the neuronal fibers than the traditional OCTs [6]. Additionally, L. M. Otto et al. presented a polarization interferometry based on the surface plasmon resonance and achieved the reflective index sensing function [7]. With the introduction of PSA, the performance of the optical systems could be significantly enhanced. In general, the conventional PSA is achieved by illuminating the sample with different polarization states, interacting the reflected/scattered light with the reference polarized light and analyzing the polarization dependent responses. However, in the conventional PSA systems, the polarizing and spectral analyzing functions are realized by the applications of extra volume or in-fiber polarizers and the grating-based instruments. These separated modules have slowed down the interrogation speed, cost inevitable loss of 
energy, complicated the system structure and made it susceptible to mechanical excitation. To overcome these drawbacks and further promote the system performance and broaden the application fields of PSA, it is of great significance to combine the polarizing and spectral analyzing and realize the functions with a single integrated component, of which the key element is the fiberbased polarization dependent diffraction grating.

In our previous work, we have proposed an in-fiber diffraction grating based on the radiant tilted fiber grating (RTFG) with $45^{\circ}$ tilt angle, which could realize the polarizing and angular dispersion functions simultaneously [8]. The in-fiber diffraction grating could achieve the perfect polarization characteristics with a degree of polarization (DOP) over 0.99 and the angular dispersion is comparable to the traditional volume diffraction grating. This unique property makes it an ideal solution for the performance promotion of the PSA. The previous works have demonstrated that the RTFG could be used as a dispersive element which has been applied in spectrally encoded imaging, OCT and wireless optical communication systems [9-14]. In this paper, we proposed a compact linear polarization spectrometer based on the modified in-fiber polarization dependent diffraction grating, which has a performance of $0.05 \mathrm{~nm}$ spectral resolution and $115 \mathrm{~nm}$ wavelength range covering $1495-1610 \mathrm{~nm}$. The radiation mode of the applied grating has been shaped into a Gaussian profile to optimize the performance of the spectrometer. Due to the fact that there is no mechanically moving element, the spectrometer has a good structural stability and high-speed dynamic spectrum acquisition ability. Besides, the spectrometer could directly analyze the polarization spectrum information without any extra polarizing components, which could significantly reduce the cost and the structural complexity of the polarization interrogation system, and further benefit the integration of the PSA systems.

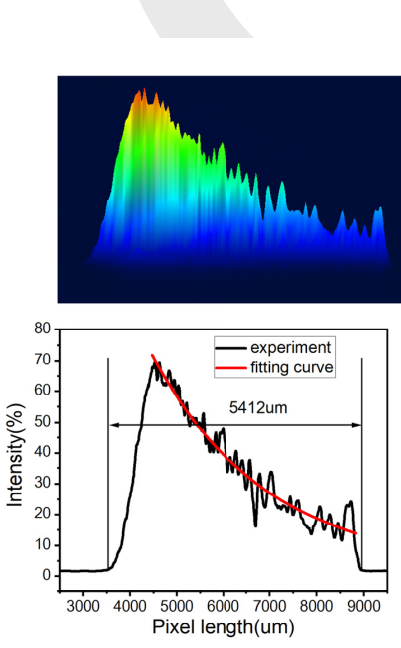

(a)

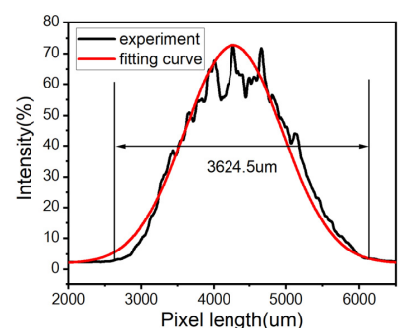

(b)
Fig. 1. The captured images and the profiles of the radiation mode of (a) uniform and (b) modified in-fiber diffraction grating;

The in-fiber diffraction grating is a perfect in-fiber dispersion device that could effectively couple the light out of fiber. However, there are some drawbacks of the radiation mode distribution of the grating. Due to the constant index modulation, the energy distribution of the radiation mode of a uniform in-fiber diffraction grating is exponentially reduced along the fiber grating [15]. This asymmetric intensity distribution makes the concentrated spot of the diffracted light become non-centrosymmetric, where the peak would diverge from the desired position and induce the non-linear response between the position of the spot and the changing of the wavelength. This non-linear response would affect the wavelength precision of the spectrometer and thus, the extra calibration is necessary. In addition, it is worth mentioning that, because of its unconcentrated energy distribution, the length of the radiation mode of uniform grating has been enlarged by its decreasing tail, which would result in the system performance degradation because the size of the focused spot is interrelated to the length of the excited radiation mode. An effective method to solve the above problem is to reduce the length of the grating, however, the radiation intensity and the energy efficiency of the grating would also be affected, which would cause the detection noise and result in the impact on the signal to noise ratio. The reduction of the grating length would limit the detection ability of the system and increase the system budget. In addition, this solution could not address the non-linear response introduced by the asymmetric distribution, which makes it not an ideal solution to this problem.

A more feasible solution is to shape the asymmetric profile to a centrosymmetric profile, where we chose the Gaussian profile in this paper. Gaussian distribution has a symmetric profile where the energy concentrates at the center and it is easy to analyze the characteristics of the transformed Gaussian beam. In our previous research [15], the radiation mode distribution is related to the profile of the refractive index modulation of the in-fiber diffraction grating. Based on this, we designed an apodized amplitude mask with a Gaussian profile and applied it to the fabrication of the grating and obtained a modified in-fiber diffraction grating with a quasi-Gaussian radiation mode distribution, see in Fig. 1. The patterns are captured by the beam profile and the pixel length stands for the position of the captured mode. The grating length of the modified grating is around $5 \mathrm{~mm}$. For the comparison, a uniform in-fiber diffraction grating has been fabricated under the same conditions. As it shown in Fig. 1, for the same length of $5 \mathrm{~mm}$, the effective length of the radiated light of the modified grating is about $3.624 \mathrm{~mm}$, while the length of the radiated light for the uniform grating is around $5.412 \mathrm{~mm}$. Besides, the maximum intensity of the radiation mode of the modified grating has been transferred to the center and the energy distribution became more concentrated than the uniform pattern. The deployment of the radiation mode shaping has effectively reduced the length of the radiation mode of the grating and optimized the distribution of the mode to a centrosymmetric profile, which would benefit the compression of the diffracted beam and the design of the fiberbased spectrometer.

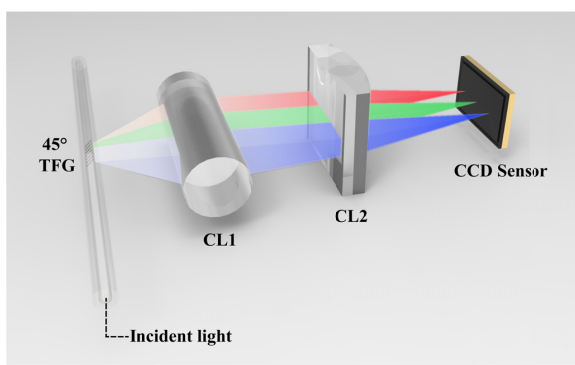

Fig. 2. Schematic diagram of the fiber optic polarization spectrometer. 
Based on the modified in-fiber diffraction grating, we built a compact linear polarization spectrometer. The schematic diagram of the spectrometer is shown in Fig. 2. As it shown in Fig. 2, the proposed spectrometer has a very straightforward structure, where the applied in-fiber diffraction grating has about $3 \mathrm{~dB}$ polarization extinction ratio (PER), $748 \mathrm{~nm}$ grating period, $1554 \mathrm{~nm}$ central wavelength and $0.054^{\circ} / \mathrm{nm}$ angular dispersion. The diffracted s-polarized light is firstly collimated and compressed by cylindrical lens (CL) 1 in perpendicular direction and further collected and focused onto CCD array (2048 pixels with 5.5um pixel dimension) by CL2. The focal length of CL1 and CL2 are $20 \mathrm{~mm}$ and $150 \mathrm{~mm}$, respectively. The diffracted light with different wavelength components would be separated into different angles and occupy different positions of CCD array. Due to the fact that the light diffracted by the grating is perfectly linear polarized, the spectrometer could implement the polarizing and spectral analysis functions simultaneously and eventually realize the polarization dependent spectrum analysis function. According to the Rayleigh criterion, the resolution of the spectrometer could be written as:

$$
\delta \lambda=\frac{\lambda^{3} f \sqrt{1+\left(1-\frac{\sqrt{2} n \Lambda}{\lambda}\right)^{2}\left(1-n^{2}\right)}}{\sqrt{2} n^{2} \Lambda D d-\lambda^{2} f \sqrt{1+\left(1-\frac{\sqrt{2} n \Lambda}{\lambda}\right)^{2}\left(1-n^{2}\right)}}
$$

Here, $f$ is the focal length of the lens, $D$ is the length of the grating and $d$ is the distance between the grating and lens, $n$ is the refractive index of fiber core, $\Lambda$ is the period of grating and $\lambda$ is the wavelength of the incident light. The performance of the spectrometer could be improved by increasing the distance between the grating and CCD sensor and using the lens with longer focal length. Theoretically, the spectrometer could be optimized to have a resolution of $0.02 \mathrm{~nm}$. And the responding wavelength range is inversely proportional to the resolution.
The resolution and the wavelength range are two of the most important parameters of spectrometric instruments. To evaluate the performance of the proposed spectrometer, we have analyzed the profiles of the focused radiation modes of the modified and uniform in-fiber diffraction gratings, which are shown in Fig. 3a. In the experiment, a single wavelength laser with the output wavelength of $1550 \mathrm{~nm}$ has been used as the light source. As illustrated in Fig. 3a, the radiation mode of the modified grating has been compressed into a small light spot with a full width at half maximum (FWHM) of 33um, while the FWHM of the uniform grating is $44 \mathrm{um}$. With the smaller focused spot, the system could achieve a higher spectral resolution, which has further verified the significance of the mode shaping. It is worth mentioning that the peak of the focused spot of the uniform grating is not located at the center as expected, which results in a non-uniform response between the wavelength and CCD signal and causes the deviation of the wavelength interrogation and the system errors.

Additionally, we have tuned the output wavelength of the laser to evaluate the performance of the spectrometer quantitatively. As it shown in Fig. 3b, the focused spots are linearly moving along the CCD array with the change of wavelength. The movement is around $2.90 \mathrm{~mm}$ for $30 \mathrm{~nm}$ wavelength change. The calibration of the wavelength and CCD pixels are plotted in Fig. 3c, which shows that the wavelength and the pixel position of CCD array is in perfect linear relationship with a sensitivity of $96.76 \mathrm{um} / \mathrm{nm}$. Consequently, based on the presented setup, the resolution and wavelength responding range of the spectrometer could achieve around $0.057 \mathrm{~nm}$ and $117 \mathrm{~nm}$ covering from $1495 \mathrm{~nm}$ to $1612 \mathrm{~nm}$, respectively. The range would become smaller for the grating with the larger angular dispersion. Moreover, as a proof of concept, we preliminarily investigated the polarization property of the spectrometer with a polarized incident light at $1565 \mathrm{~nm}$, see in Fig. $3 \mathrm{~d}$. The intensity of the measured spectra has an $86.7 \%$ intensity difference with two different polarization states, which has verified the strong polarization dependency and the feasibility of polarization spectrum analysis of the spectrometer.
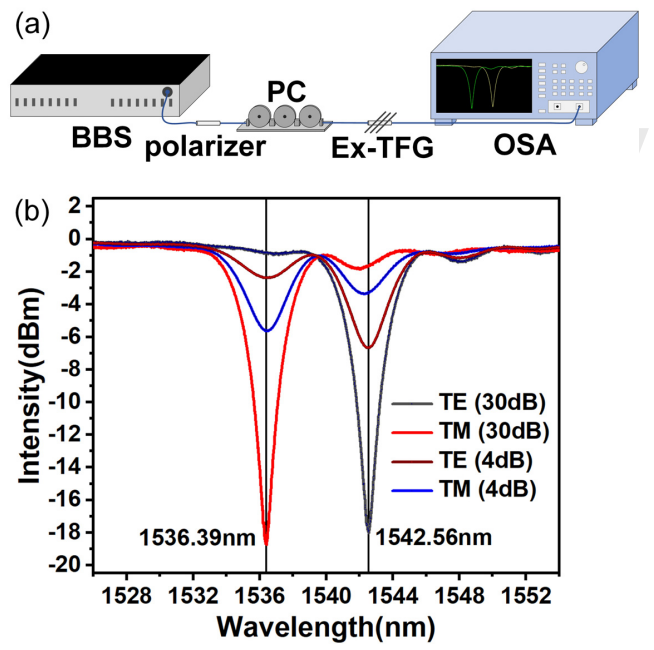

Fig. 4. (a) the experimental setups and (b) the spectrums of Ex-TFG obtained by OSA with different PER (30dB and $4 \mathrm{~dB}$ ) polarizers.

For the traditional polarization spectrum measurement system, the applications of volume or in-fiber polarizers are necessary to
Fig. 3. (a) the captured image (inserted) and the intensity profile of the focused radiated light spot of modified and uniform in-fiber diffraction gratings; (b) the spot positions versus different wavelengths; (c) relationship between the wavelength and pixel of CCD; (d) the spectra of tuneable laser with different polarization states at $1565 \mathrm{~nm}$.
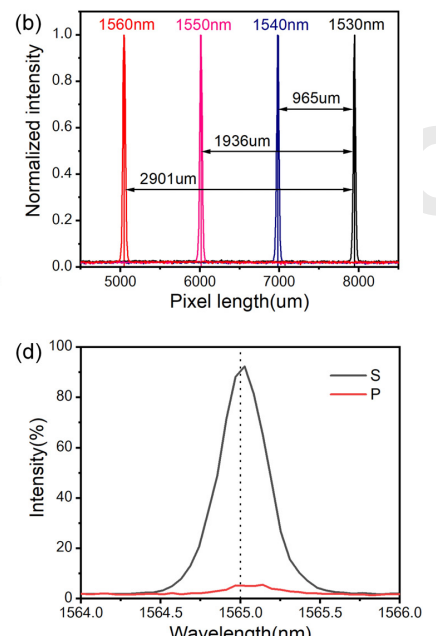
generate and detect the linearly polarized light. Unfortunately, the result of the measurement has a dependency on the performance of the applied polarization devices. To verify this, we evaluated the performance of the spectrometer and compared with the traditional measurement method. In the experiment, we measured the polarization transmission spectra of an excessively tilted fiber grating (Ex-TFG), which has a pair of orthogonal polarization transmission peaks defined as TE and TM modes [16]. Fig. 4a shows the traditional experimental setup which consists of broadband light source (BBS), polarizers, polarization controller (PC) and optical spectrum analyzer. The PC is used to adjust the polarization state of the incident light to obtain different polarization resonant peaks, where the dual peaks would appear and suppress alternatively. In the experiment, we compared the results measured by two different PER polarizers ( $4 \mathrm{~dB}$ and $30 \mathrm{~dB}$ ), respectively. Since the polarization spectra of the Ex-TFG is strongly dependent on the degree of polarization of incident light, the TM and TE modes of Ex-TFG measured by two polarizers have the same peak wavelengths of $1536.39 \mathrm{~nm}$ and $1542.56 \mathrm{~nm}$, respectively, but with different peak intensity. The results measured with the $4 \mathrm{~dB}$ polarizer has about $12 \mathrm{~dB}$ peak intensity difference compared with the results measured with the $30 \mathrm{~dB}$ polarizer. The difference of the measurement results indicates that the performance of the traditional polarization analysis system would be affected by the applied polarizers.

(a)

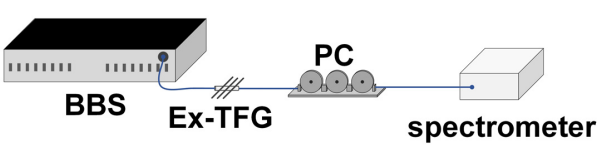

(b)

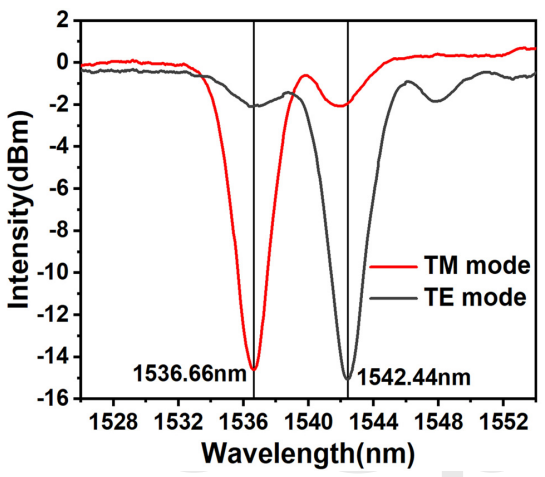

Fig. 5. (a) the experimental setups and (b) the spectrums of Ex-TFG obtained by the polarization-related spectrometer.

Furthermore, we have measured the transmission spectrum of Ex-TFG by using the proposed polarization-related spectrometer, and the results are shown in Figure 5. As it shown in Fig. 5a, the new measuring setup doesn't need a polarizer anymore. In the experiment, we measured the same Ex-TFG with the proposed spectrometer under the same conditions as the previous one. The proposed spectrometer is immune to the performance degradation effect of the polarizers because the light diffracted by the grating is almost linearly polarized with an extraordinarily high DOP, see in Fig. 5b. Although the PER of the applied in-fiber diffraction grating is only around $3 \mathrm{~dB}$ in this setup, it could achieve the comparable results with the $30 \mathrm{~dB}$ polarizers. The wavelength consistencies between these two systems only have around
$0.27 \mathrm{~nm}$ and $0.12 \mathrm{~nm}$ differences for the TM and TE cladding modes, respectively, which might be caused by the slightly nonlinear wavelength-pixel relationship of the spectrometer. Finally, we have demonstrated a single polarization spectrometer with perfect performance of compact structure, high resolution, good wavelength consistency and great polarization characteristics.

In conclusion, we have proposed a linear polarization spectrometer based on the radiation mode shaped modified infiber diffraction grating. The spectrometer has a good performance with a simple structure, which is comparable to the commercial OSA according to the experimental results. The spectrometer has integrated the functions of polarizing and spectral analysis, which achieve the polarization spectrum analysis function without any applications of polarizers in the system, and obtain similar results to the high PER polarizers. The performance of the spectrometer could be further enhanced by optimizing the system structure, using better CCDs and redesigning the in-fiber diffraction grating to achieve better dispersion characteristics. The proposed spectrometer would promote the developments and applications the fiber-based devices in polarization-related spectral analysis, spectral imaging, fiber sensing and fiber communication fields.

Funding. National Key Research and Development Program of China (2018YFB2100902); Major Projects of Technical Innovation of Hubei (2019AAA053 and 2018AAA040); National Natural Science Foundation of China (NSFC) (61505244); Science Fund for Creative Research Groups of the Nature Science Foundation of Hubei (2018CFA004).

\section{References}

1. F. Liu, P. Han, Y. Wei, K. Yang, S. Huang, X. Li, G. Zhang, L. Bai, and X. Shao, Opt. Lett. 43, 4903-4906 (2018).

2. T. Schmoll, E. Gotzinger, M. Pircher, C. K. Hitzenberger, and R. A. Leitgeb, Opt. Lett. 35, 241-243 (2010).

3. K. H. Kim, B. H. Park, Y. Tu, T. Hasan, B. Lee, J. Li, and J. F. de Boer, Opt. Express 19, 552-561 (2011).

4. M. Zhao and J. A. Izatt, Opt. Lett. 34, 205-207 (2009).

5. E. Gotzinger, M. Pircher, and C. K. Hitzenberger, Opt. Express 13, 1021710229 (2005).

6. H. Wang, T. Akkin, C. Magnain, R. Wang, J. Dubb, W. J. Kostis, M. A. Yaseen, A. Cramer, S. Sakadzic, and D. Boas, Opt. Lett. 41, 2213-2216 (2016).

7. L. M. Otto, D. A. Mohr, T. W. Johnson, S. H. Oh, and N. C. Lindquist, Nanoscale, 7, 4226-4233 (2015)

8. H. Qin, Q. He, Z. Xing, X. Guo, Z. Yan, Q. Sun, C. Wang, K. Zhou, D. Liu, and L. Zhang, Opt. Lett. to be published.

9. J. L. Wagener, T. A. Strasser, J. R. Pedrazzani, J. DeMarco, and D. DiGiovanni, in 23rd European Conference on Optical Communication, Conf. Publ. 448, 65-68 (1997).

10. K. Feder, P. Westbrook, J. Ging, P. Reyes, and G. Carver, IEEE. Photonics Technol. Lett. 15, 933-935 (2003).

11. K. Zhou, X. Cheng, Z. Yan, A. Adedotun, and L. Zhang, in Bragg Gratings, Photosensitivity, and Poling in Glass Waveguides (Optical Society of America 2012), BW2E. 7.

12. G. Wang, C. Wang, Z. Yan, and L. Zhang, Opt. Lett. 41, 2398-2401 (2016).

13. S. Remund, A. Bossen, X. Chen, L. Wang, L. Zhang, B. Považay, and C. Meier, Proc. SPIE 9129, 91293G (2014).

14. G. Wang, U. Habib, Z. Yan, N. J. Gomes, Q. Sui, J. Wang, L. Zhang, and C. Wang, J. Lightwave Technol. 36, 4618-4625 (2018).

15. H. Qin, Q. He, Z. Xing, X. Guo, Z. Yan, Q. Sun, K. Zhou, H. Wang, D. Liu, and L. Zhang, J. Lightwave Technol. 37, 3777-3783 (2019).

16. Z. Yan, H. Wang, C. Wang, Z. Sun, G. Yin, K. Zhou, Y. Wang, W. Zhao, and L. Zhang, Opt. Express 24, 12107-12115 (2016). 
1. F. Liu, P. L. Han, Y. Wei, K. Yang, S. Z. Huang, X. Li, G. Zhang, L. Bai, and X. $P$. Shao, "Deeply seeing through highly turbid water by active polarization imaging," Opt Lett 43, 4903-4906 (2018).

2. T. Schmoll, E. Gotzinger, M. Pircher, C. K. Hitzenberger, and R. A. Leitgeb, "Single-camera polarization-sensitive spectral-domain OCT by spatial frequency encoding," Opt Lett 35, 241-243 (2010).

3. K. H. Kim, B. H. Park, Y. Tu, T. Hasan, B. Lee, J. Li, and J. F. de Boer, "Polarization-sensitive optical frequency domain imaging based on unpolarized light," Opt Express 19, 552-561 (2011).

4. M. Zhao and J. A. Izatt, "Single-camera sequential-scan-based polarization-sensitive SDOCT for retinal imaging," Opt Lett 34, 205-207 (2009).

5. E. Gotzinger, M. Pircher, and C. K. Hitzenberger, "High speed spectral domain polarization sensitive optical coherence tomography of the human retina," Opt. Express 13, 10217-10229 (2005).

6. H. Wang, T. Akkin, C. Magnain, R. Wang, J. Dubb, W. J. Kostis, M. A. Yaseen, A. Cramer, S. Sakadzic, and D. Boas, "Polarization sensitive optical coherence microscopy for brain imaging," Opt Lett 41, 2213-2216 (2016).

7. L. M. Otto, D. A. Mohr, T. W. Johnson, S. H. Oh, and N. C. Lindquist, "Polarization interferometry for real-time spectroscopic plasmonic sensing," Nanoscale 7, 4226-4233 (2015).

8. H. Qin, Q. He, Z. Xing, X. Guo, Z. Yan, Q. Sun, C. Wang, K. Zhou, D. Liu, and L. Zhang, "In-fiber Single Polarization Diffraction Grating based on Radiant Tilted Fiber Grating," Opt. Lett. to be published.

9. J. L. Wagener, T. A. Strasser, J. R. Pedrazzani, J. DeMarco, and D. DiGiovanni, "Fiber grating optical spectrum analyzer tap," in Integrated Optics and Optical Fibre Communications, 11th International Conference on, and 23rd European Conference on Optical Communications (Conf. Publ. No.: 448), (IET, 1997), 65-68.

10. K. Feder, P. Westbrook, J. Ging, P. Reyes, and G. Carver, "In-fiber spectrometer using tilted fiber gratings," IEEE Photonics Technology Letters 15, 933-935 (2003).

11. K. Zhou, X. Cheng, Z. Yan, A. Adedotun, and L. Zhang, "Optical Spectrum Analyzer using a $45 \bigcirc$ tilted fiber grating," in Bragg Gratings, Photosensitivity, and Poling in Glass Waveguides, (Optical Society of America, 2012), BW2E. 7.

12. G. Wang, C. Wang, Z. Yan, and L. Zhang, "Highly efficient spectrally encoded imaging using a $45^{\circ}$ tilted fiber grating," Opt Lett 41, 2398-2401 (2016).

13. S. Remund, A. Bossen, X. Chen, L. Wang, L. Zhang, B. Považay, and C. Meier, "Fiber optically integrated cost-effective spectrometer for optical coherence tomography," in Biophotonics: Photonic Solutions for Better Health Care IV, (International Society for Optics and Photonics, 2014), 91293G.

14. G. Wang, U. Habib, Z. Yan, N. J. Gomes, Q. Sui, J. Wang, L. Zhang, and C. Wang, "Highly efficient optical beam steering using an in-fiber diffraction grating for full duplex indoor optical wireless communication," J. Lightwave Technol. 36, 4618-4625 (2018).

15. H. Qin, Q. He, Z. Xing, X. Guo, Z. Yan, Q. Sun, K. Zhou, H. Wang, D. Liu, and L. Zhang, "Numerical and Experimental Characterization of Radiation Mode of $45^{\circ}$ Tilted Fiber Grating," J. Lightwave Technol. 37, 3777 - 3783 (2019). (2019).

16. Z. Yan, H. Wang, C. Wang, Z. Sun, G. Yin, K. Zhou, Y. Wang, W. Zhao, and L. Zhang, "Theoretical and experimental analysis of excessively tilted fiber gratings," Opt. Express 24, 12107-12115 (2016). 